\title{
Associazione tra composti volatili solforati, malattia parodontale e reflusso gastroesofageo in pazienti con alitosi
}

\section{Association between volatile sulphur compounds, periodontal disease and gastroesophageal reflux disease in patients with halitosis}

Ricevuto il

20 luglio 2013

Accettato il

5 dicembre 2013

*Autore di riferimento

Davide Testi

davide_testi@hotmail.it
RIASSUNTO

OBIETTIVI. Lo scopo della ricerca è stato quello di valutare la correlazione fra tre diversi composti volatili solforati (Volatile Sulphur Compounds, VSC), la presenza di patologie parodontali e il reflusso gastroesofageo.

MATERIALI E METODI. Per lo studio è stato utilizzato un gascromatografo, che consente di misurare i VSC singolarmente e di visualizzarli in un diagramma secondo la loro concentrazione espressa in nanogrammi/10 mL. Quaranta pazienti sono stati suddivisi in quattro gruppi in base alla presenza di patologie parodontali e al riferito sintomo di reflusso gastroesofageo.

RISULTATI. I risultati hanno evidenziato come la condizione di malattia parodontale sia correlata a un'alta concentrazione di solfuro di idrogeno e il reflusso gastroesofageo sia associato alla concentrazione di dimetilsolfuro.

CONCLUSIONI. L'alitosi è una condizione caratterizzata dalla produzione di alito maleodorante che può indicare una serie di malattie sistemiche e locali o specifiche abitudini alimentari e può essere fattore diagnostico di patologie psichiatriche. L'impiego del gascromatografo aiuta a stabilire la presenza di gas solforati nell'alito e offre dati aggiuntivi per la diagnosi di malattia parodontale e di reflusso gastroesofageo.

\section{PAROLE CHIAVE}

- Alitosi

- Gascromatografo

- Composti volatili solforati (VSC)

- Reflusso

- Parodontite 


\section{ABSTRACT}

OBJECTIVES. The aim of this research was to evaluate the correlation between three different kinds of Volatile Sulphur Compounds (VSC), periodontal disease and gastroesophageal reflux disease.

MATERIALS AND METHODS. In this study, we used a gas chromatograph capable of measuring levels of VSCs individually and displaying their concentration in a diagram expressed in nanograms/10 $m L .40$ patients were selected on the basis of diagnosed periodontal diseases and the reported symptom of gastroesophageal reflux.

RESULTS. Our results show that periodontal disease was linked to a high concentration of hydrogen sulphide, whereas gastroesophageal reflux was linked to high concentration of dimethyl sulfide.

CONCLUSIONS. Halitosis is a condition characterized by consistent foul-smelling breath that may indicate a wide range of local and systemic diseases, or may be due to specific eating habits. It may also be a diagnostic factor for psychiatric disorders. A gas chromatograph detecting sulphur gases in breath makes the diagnosis of halitosis easier and adds useful data for the diagnosis of periodontal disease and gastroesophageal reflux disease.

\section{KEY WORDS}

\section{- Halitosis}

- Gas chromatograph

- Volatile sulphur compounds (VSC)

- Reflux

- Periodontitis

\section{INTRODUZIONE}

L'alitosi è una condizione caratterizzata dalla produzione di alito maleodorante che è talvolta percepibile dalle persone che ci circondano o da noi stessi. L'alitosi può indicare una vasta serie di malattie sistemiche e locali, abitudini alimentari, patologie a carico del cavo orale e nondimeno può essere fattore diagnostico di alcune patologie psichiatriche; la percezione su se stessi di tale disturbo può rappresentare inoltre un fattore di imbarazzo sociale.

La percezione organolettica sgradevole è determinata dalla concentrazione di composti volatili solforati (Volatile Sulphur Compounds, VSC) che determinano il cattivo odore perché contengono un'alta concentrazione di gruppi solfuri.

Fin dai primi anni Settanta alcuni studi

[1] hanno identificato tre tipologie di VSC responsabili di alitosi:

- il solfuro di idrogeno $\left(\mathrm{H}_{2} \mathrm{~S}\right)$;

- il metilmercaptano $\left(\mathrm{CH}_{3} \mathrm{SH}\right)$;

- il dimetilsolfuro $\left(\mathrm{CH}_{3}\right)_{2} \mathrm{~S}$.

Questi tre tipi di gas hanno origine da diversi substrati e possono essere presenti insieme o separatamente nel cavo orale. Nello specifico, i VSC prodotti dai substrati dell'aminoacido cisteina presentano un'elevata concentrazione di solfuro di idrogeno, mentre i VSC prodotti dai substrati della metionina presentano un'alta concentrazione di metilmercaptano.

Il reflusso gastroesofageo è una condizione patologica nella quale, a causa della lassità dello sfintere cardiale, il contenuto dello stomaco risale periodicamente l'esofago provocando danni alle strutture dentarie e ai tessuti del cavo orale, oltre che all'esofago stesso.

\subsection{CLASSIFICAZIONE DELL'ALITOSI}

Diversi studi risalenti al decennio scorso hanno contribuito alla classificazione dell'alitosi. Di questi, uno in particolare ha classificato l'alitosi in modo ampio e completo [2].

L'alitosi può essere suddivisa in diverse categorie: alitosi genuina, pseudoalitosi e alitofobia.

\section{$>$ Alitosi genuina}

Per alitosi genuina si intende un livello di percezione del cattivo odore superiore alla tollerabilità socialmente accettabile ed è suddivisa a sua volta in alitosi fisiologica e patologica.

Alitosi fisiologica. L'alitosi fisiologica che ha origine dal cavo orale è causata principalmente dalla dieta, dalla secchezza orale, dal fumo e dalla presenza di protesi o restauri incongrui.

- Dieta. Quando l'igiene orale domiciliare non è corretta e costante, la sola azione della lingua e delle labbra non è sufficiente a garantire la rimozione dei residui di cibo che, rimanendo nel cavo orale, si decompongono e comportano tale tipologia di alitosi. I principali cibi alitogeni sono: cipolla, aglio, spezie esotiche, alcuni formaggi, il pesce e il caffè. È inoltre opportuno sottolineare che una dieta ricca di proteine favorisce l'alitosi poiché consente ai batteri di usufruire di una maggiore quantità di substrato proteico.

- Secchezza orale. L'alitosi può essere causata anche da xerostomia transitoria poiché in mancanza dell'azione detergente della saliva aumenta il 
substrato disponibile con il quale i batteri producono i gas solfurei.

- Prodotti della nicotina. L'utilizzo di sigarette, pipa, sigari, tabacco da fiuto e da masticare causa alitosi e irrita i tessuti gengivali.

- Protesi e restauri incongrui. Talvolta le protesi totali, parziali o i restauri incongrui possono comportare un eccessivo accumulo di placca e di residui di cibo, dalla cui putrefazione si liberano i gas VSC.

Alitosi patologica. L'alitosi patologica è suddivisa in alitosi con cause orali ed extraorali.

- Cause orali. L'alitosi da cause orali è determinata da processi patologici del cavo orale quali parodontite aggressiva, parodontite ulcero-necrotica e xerostomia da ipofunzione delle ghiandole salivari maggiori e/o minori, come per esempio nel caso di pazienti con sindrome di Sjögren. Nelle parodontopatie si ha un incremento dei gas VSC in quanto aumentando i batteri dei complessi arancione e rosso si assiste a una maggiore catabolisi e degradazione dei substrati proteici.

- Cause extraorali. L'alitosi da cause extraorali è determinata da patologie del tratto nasale, paranasale (sinusiti croniche), respiratorio (bronchiti croniche), faringeo (infezioni della gola, faringiti, tonsilliti), gastrointestinale (reflusso gastroesofageo) e in minor parte da cause sistemiche (epatopatie gravi, insufficienza renale cronica, intolleranza al lattosio e assunzione di farmaci antistaminici, antidepressivi, diuretici e ansiolitici).

\section{> Pseudoalitosi e alitofobia}

La pseudoalitosi e l'alitofobia sono patologie psicologiche e si distinguono in base alla possibilità dell'operatore di convincere o meno il paziente della non obiettività della sua percezione. In entrambi i casi non esiste una diagnosi di evidente alitosi $[3,4]$.

\subsection{REAZIONE CATABOLICA}

Le principali reazioni chimiche che determinano la formazione dei VSC si basano sulla catabolisi della cisteina e della metionina.

La L-cisteina viene attivata dal catalizzatore L-cisteina desulidrasi dei batteri Gram-negativi che permette la produzione di 2-chetopropanato, solfuro di idrogeno e $\mathrm{NH}_{4}$.

L'enzima batterico L-metionina-liasi catalizza la L-metionina in 2-chetobutirrato (usato dai batteri come fonte di energia), metilmercaptano (metanetiolo) e $\mathrm{NH}_{4}$. Le proteine che vengono degradate derivano dai cibi, dalla saliva e dai cheratinociti del cavo orale.

I batteri generalmente coinvolti nelle reazioni cataboliche della cisteina e della metionina sono batteri anaerobi Gramnegativi, compresi i principali patogeni parodontali $[5,6]$.

La produzione in larga quantità dei composti a base di zolfo nell'area parodontale ha il potere di esercitare effetti distruttivi sui tessuti della mucosa orale, specialmente sul tessuto connettivo; i VSC infatti accrescono la permeabilità della membrana parodontale, facilitando così l'ingresso delle tossine batteriche $\mathrm{e}$ degli stessi batteri nella barriera epiteliale, causando la rottura del collagene. Di particolare importanza sono la soppressione della sintesi del collagene e l'aumento della sua degradazione nelle colture di fibroblasti gengivali esposte al metilmercaptano, colture che evidenziano il 70\% in meno di crescita rispetto alla norma [7].
I gas, inoltre, stimolano la produzione di specifiche citochine in sistemi di coltura, il che denota la loro capacità di attivare la risposta immunitaria del sistema, provocando perciò un aumento nella produzione e nell'attivazione di enzimi collagenolitici.

In alcuni studi si è osservato che i pazienti affetti da paradontopatie producono più VSC rispetto alla norma $[8,9]$; è stata infatti dimostrata la correlazione positiva tra concentrazioni di VSC, stato parodontale e placca presente sul dorso della lingua. In taluni lavori molto recenti [10-12] si correla l'aumento dei gas VSC (solfuro di idrogeno, metilmercaptano e dimetilsolfuro) al peggioramento della malattia parodontale.

Lo scopo del presente lavoro è valutare la correlazione fra i tre diversi gas VSC, la presenza di malattia parodontale e il reflusso gastroesofageo.

\section{MATERIALI E METODI}

\subsection{ANALISI DEI GAS VSC}

Per questo studio osservazionale è stato utilizzato un gascromatografo (Oral Chroma $^{\mathrm{TM}}$, Abimedical) che consente di misurare i gas VSC singolarmente e di visualizzarli in un diagramma secondo la loro concentrazione espressa in nanogrammi/10 $\mathrm{mL}$. Le soglie di percettibilità organolettica indicate dalla casa produttrice del macchinario sono: $1,5 \mathrm{ng} / 10$ $\mathrm{mL}$ di solfuro di idrogeno; $0,49 \mathrm{ng} / 10$ $\mathrm{mL}$ di metilmercaptano; $1,9 \mathrm{ng} / 10 \mathrm{~mL}$ di dimetilsolfuro.

\subsection{CRITERI DI INCLUSIONE}

Sono stati inclusi i pazienti che si sono presentati alla UOC Odontostomatologia dell'Ospedale San Giovanni Calibita Fatebenefratelli di Roma a causa di riferiti 
problemi di alitosi, in assenza di malattie sistemiche e di terapie farmacologiche. Alla prima visita sono stati raccolti i seguenti dati e materiali: questionario anamnestico, radiografia ortopantomografica (e/o esame endorale completo), fotografie cliniche, indici parodontali (profondità di sondaggio, $\mathrm{PD}$; sanguinamento al sondaggio, BOP), prelievo dell'alito.

\subsection{RICERCA}

La ricerca ha incluso pazienti, 20 donne e 20 uomini, con età media di 42 anni.

I pazienti sono stati suddivisi, in base alla presenza o assenza di patologie parodontali e in base al riferito sintomo di reflusso gastroesofageo, nel seguente modo:

- 10 casi di pazienti con malattia parodontale e reflusso (I gruppo);

- 10 casi di pazienti con malattia parodontale senza reflusso (II gruppo);

- 10 casi di pazienti sani con reflusso (III gruppo);

- 10 casi di pazienti sani senza reflusso (IV gruppo).

La presenza di malattia parodontale è intesa come PD > $3 \mathrm{~mm}$ e presenza di sanguinamento al sondaggio. L'assenza di malattia parodontale (pazienti sani) è intesa come PD $<3$ mm e assenza di sanguinamento al sondaggio. La presenza di reflusso gastroesofageo è stata accertata mediante visita specialistica

\subsection{PRELIEVO E PREPARAZIONE DEL TEST}

Dopo l'inserimento di una siringa da 2 $\mathrm{mL}$ nel cavo orale (circa $2 \mathrm{~cm}$ davanti l'ugola) è stato chiesto a ogni paziente di tenere la bocca chiusa e di respirare con il naso per circa 30 secondi, evitando di toccare con la lingua la punta della siringa.

Si è poi proceduto ad aspirare l'aria dal cavo orale, svuotando la siringa interamente e riaspirando l'aria per un totale di $0,5 \mathrm{~mL}$.

Questi $0,5 \mathrm{~mL}$ di aria sono stati inseriti, tramite apposito ago applicato alla suddetta siringa, nel gascromatografo che in circa 8 minuti ha determinato le concentrazioni dei tre gas VSC nei campioni e ha elaborato un diagramma.

Gli autori hanno ottenuto il consenso informato da parte dei pazienti che si sono sottoposti al test. Lo studio è stato realizzato in accordo con gli standard etici stabiliti nella Dichiarazione di Helsinki del 1964.

\section{RISULTATI}

I risultati ottenuti, espressi come media con deviazione standard su 10 pazienti a gruppo, hanno permesso di evidenziare quanto segue.

\section{N. 20 pazienti con malattia parodontale}

- 10 pazienti con reflusso (I gruppo): quantità media di solfuro di idrogeno $1,97 \pm 0,773 \mathrm{ng} / 10 \mathrm{~mL}$; metilmercaptano 0,52 \pm 0,344 ng/10 mL; dimetilsolfuro $2,71 \pm 1,370 \mathrm{ng} / 10 \mathrm{~mL}$.

- 10 pazienti senza reflusso (II gruppo): quantità media di solfuro di idrogeno $1,77 \pm 0,449 \mathrm{ng} / 10 \mathrm{~mL}$; metilmercaptano $0,84 \pm 0,731 \mathrm{ng} / 10 \mathrm{~mL}$; dimetilsolfuro $0,97 \pm 0,529 \mathrm{ng} / 10 \mathrm{~mL}$.

\section{N. 20 pazienti sani}

- 10 pazienti con reflusso (III gruppo): quantità media di solfuro di idrogeno $0,45 \pm 0,423 \mathrm{ng} / 10 \mathrm{~mL}$; metilmercaptano $0,39 \pm 0,257 \mathrm{ng} / 10 \mathrm{~mL}$; dimetilsolfuro 4,40 $\pm 1,466 \mathrm{ng} / 10 \mathrm{~mL}$.

- 10 pazienti senza reflusso (IV gruppo): quantità media di solfuro di idrogeno $0,72 \pm 0,286 \mathrm{ng} / 10 \mathrm{~mL}$; metilmercaptano 0,25 \pm 0,09 ng/10 mL;

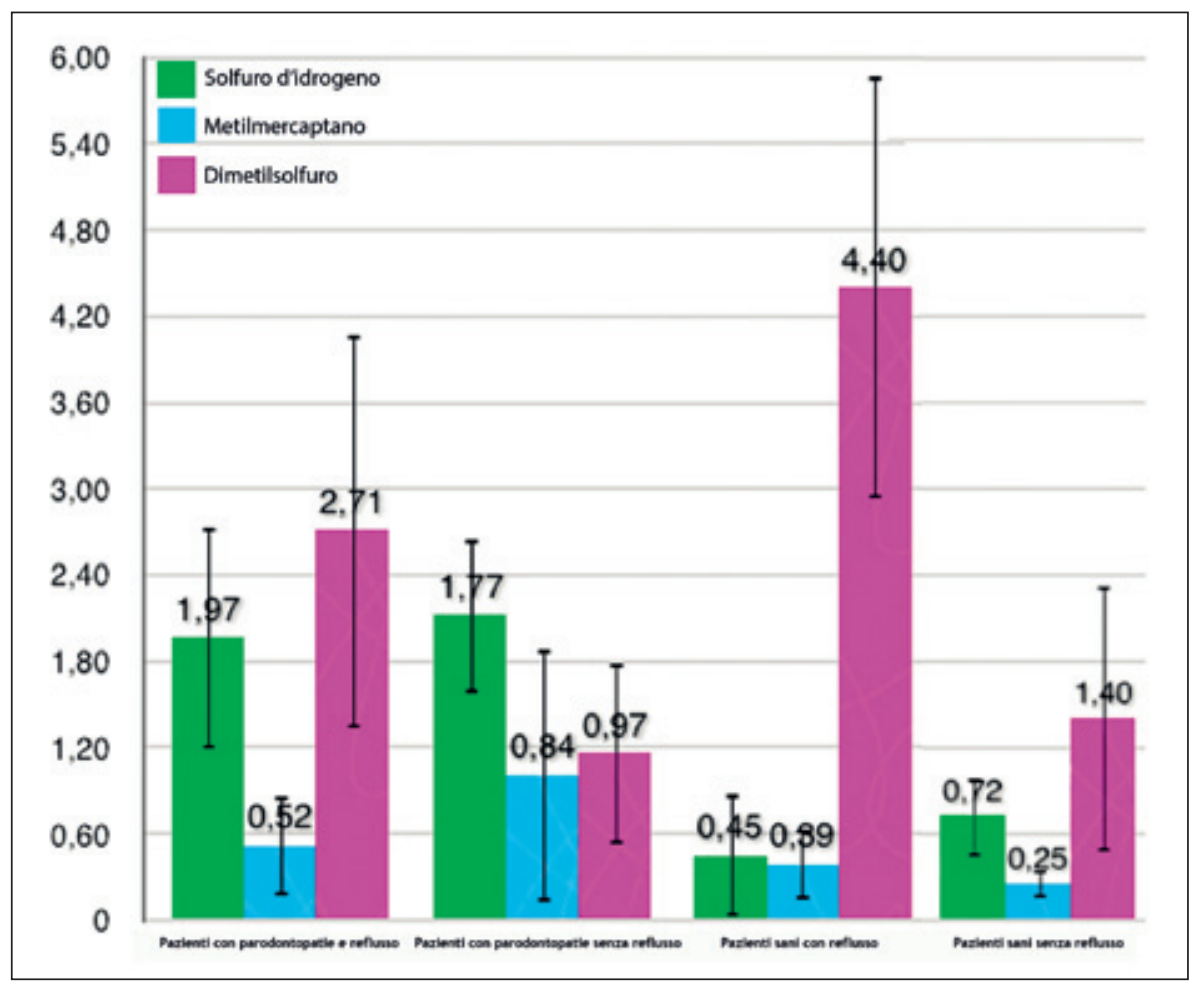

Fig. 1 Valori medi dei gas VSC 
dimetilsolfuro $1,40 \pm 0,928 \mathrm{ng} / 10 \mathrm{~mL}$ (fig. 1).

\section{CONCLUSIONI}

La presenza di gas solforati nell'aria espirata da pazienti con alitosi è un dato certo; il nostro interesse si è concentrato sui range entro i quali i gas presenti possono o meno essere considerati sgradevoli e sulle cause dell'aumento dei gas VSC.

L'analisi dei dati può far ipotizzare che l'alitosi correlata all'elevata presenza di solfuro di idrogeno sia direttamente legata alla malattia parodontale, e quindi alla placca batterica, e che l'alitosi correlata all'elevata presenza di dimetilsolfuro sia legata al reflusso gastroesofageo.

Questi risultati, in linea con gli studi citati, confermano che la presenza di solfuro di idrogeno nell'alito dei pazienti è causato da reazioni cataboliche dei batteri del biofilm parodontale; per questo gruppo di pazienti la terapia causale è il trattamento più efficace.

Nel caso di pazienti con reflusso gastroesofageo si può soltanto supporre che il passaggio di acido nell'esofago e poi nel cavo orale produca una degradazione dell'epitelio o ne indebolisca le difese naturali, rendendolo più suscettibile ai batteri patogeni. Il ruolo dell'odontoiatra per questi pazienti si limita alla diagnosi e il trattamento è da deman- dare al gastroenterologo. In riferimento all'analisi del metilmercaptano gli autori preferiscono analizzare un campione più congruo al caso: l'analisi della letteratura ha infatti mostrato che la formazione di metilmercaptano è provocata da condizioni non prese in considerazione nella scelta del campione di questo studio, quindi si riservano di presentare i valori di tale gas solo come risultato secondario dello studio.

Nei limiti di questo studio e considerato il basso numero di soggetti esaminati, si può concludere che la diagnosi di alitosi tramite gascromatografo aiuta a stabilire la presenza di gas solforati, suggerisce un dato attendibile nella determinazione del livello di igiene orale (con la possibilità di diagnosi e di prevenzione della malattia parodontale) e, se il dato sarà confermato da altri studi con un campione più elevato, potrebbe essere utilizzata come mezzo diagnostico di reflusso gastroesofageo.

\section{CONFLITTO DI INTERESSI}

Gli autori dichiarano di non avere alcun conflitto di interessi.

\section{FINANZIAMENTI ALLO STUDIO}

Gli autori dichiarano di non aver ricevuto finanziamenti istituzionali per il seguente studio.

\section{BIBLIOGRAFIA}

1. Tonzetich J. Direct gas chromatographic analysis of sulphur compounds in mouth air in man. Arch Oral Biol 1971;16(6):587-97.

2. Yaegaki K, Coil JM. Examination, classification, and treatment of halitosis; clinical perspectives. J Can Dent Assoc 2000;66(5): 257-61.

3. Lochner C, Stein DJ. Olfactory reference syndrome: diagnostic criteria and differential diagnosis. J Postgrad Med 2003;49(4):328-31.

4. Bohn P. Imagined halitosis: a social phobia symptom? J Calif Dent Assoc 1997;25(2):161-

5. Solis-Gaffer MC, Fischer TJ, Gaffer A. Instrumental evaluation of odor produced by specific oral microorganisms. J Soc Cosmet Chem 1979;30:241.

6. McNamara TF, Alexander JF, Lee M. The role of microorganisms in the production of oral malodor. Oral Surg Oral Med Oral Pathol 1972;34(1):41-8.

7. $\mathrm{Ng} \mathrm{W}$, Tonzetich J. Effect of hydrogen sulfide and methyl mercaptan on the permeability of oral mucosa. J Dent Res 1984;63(7):994-7.

8. Miyazaki H, Sakao S, Katoh Y, Takehara T Correlation between volatile sulphur compounds and certain oral health measurements in the general population. J Periodontol 1995;66(8):679-84.

9. Morita M, Wang HL. Association between oral malodor and adult periodontitis: a review. J Clin Periodontol 2001;28(9):813-9.

10. Makino Y, Yamaga T, Yoshihara A, Nohno $\mathrm{K}$, Miyazaki H. Association between volatile sulfur compounds and periodontal disease progression in elderly non-smokers. J Periodontol 2012;83(5):635-43.

11. Ehizele AO, Ojehanon PI. Relationship between the concentration of volatile sulphur compound and periodontal disease severity in Nigerian young adults. Niger Med J 2013;54(3):149-52.

12. Kishi M, Ohara-Nemoto $Y$, Takahashi M, Kishi K, Kimura S, Aizawa F, et al. Prediction of periodontopathic bacteria in dental plaque of periodontal healthy subjects by measurement of volatile sulfur compounds in mouth air. Arch Oral Biol 2013;58(3): 324-30. 